\title{
ESTIMATION OF CARBON EMISSION AND IDENTIFICATION OF DRIVING FACTORS IN THE CIRCUM-CHANGSHA- ZHUZHOU-XIANGTAN URBAN AGGLOMERATION OF CHINA
}

\author{
DENG, Q. Z. - TIAN, Y. C." \\ School of Business, Hunan University of Science and Technology, Xiangtan 411201, China \\ *Corresponding author \\ e-mail:819326168@qq.com
}

(Received $3^{\text {rd }}$ Apr 2019; accepted $17^{\text {th }}$ May 2019)

\begin{abstract}
Based on panel statistics on Circum-Changsha-Zhuzhou-Xiangtan urban agglomeration of China in 2008-2016, the area's carbon emissions originate from three distinct sources: agriculture, industry and transportation in a "bottom-up" way for estimation. The Logarithmic Mean Divisia Index (LMDI) model is used to identify the driving factors that influence carbon emission in this urban agglomeration. Finally, the interaction between carbon emission and economic development was scientifically evaluated based on decoupling elasticity index. The results show: (1) the carbon emission of Circum-Changsha-Zhuzhou-Xiangtan urban agglomeration increased in 2008-2011, and slightly fluctuated in 2011-2016. According to the Environmental Kuznets Curve (EKC), there is an obvious "inverted-U" curve between carbon emission and per capita Gross Domestic Product (GDP), means the carbon emission will be effectively controlled as the economic development reaches a certain level; (2) LMDI decomposition shows the economic growth and human capital played a positive role in promoting the growth and change of carbon emissions of the whole urban agglomeration, while the energy intensity and industrial structure had a negative effect; (3) the decoupling level of Circum-Changsha-ZhuzhouXiangtan urban agglomeration as a whole tends to be slightly higher than the growth rate of carbon emission, among which the core region has a weak decoupling trend while Hengyang and Changde of China repeatedly appear to be in strong decoupling state.
\end{abstract}

Keywords: green development, EKC curve, LMDI model, decoupling index, emission-reduction

\section{Introduction}

The increasing greenhouse effect drew international attention towards problems concerning the ecological environment. At the Copenhagen meeting, China promised to "reduce the carbon dioxide emission per unit GDP by $40-45 \%$ by 2020 compared to 2005." Subsequently, the State Council listed low carbon level as one of the important development goals in the Plan for Ecological Environment Protection during the Thirteenth Five-year Plan Period. In the process of accelerating the development of urbanization, the rapid concentration of industry and population makes cities become the key areas of energy conservation and emission reduction. Therefore, China has successively issued many plans for building low-carbon cities. Located in the middle reaches of the Yangtze River Economic Belt of China, the Circum-Changsha-ZhuzhouXiangtan urban agglomeration is responsible for the rise of the central region. Under the two development strategies of the Belt and Road and Circum-Changsha-ZhuzhouXiangtan Integration, the Circum-Changsha-Zhuzhou-Xiangtan urban agglomeration should set up a model among the urban agglomerations in the middle reaches of the Yangtze River as well as bear more responsibilities in national and regional development and environmental governance. In this context, scholars have produced researches on $\mathrm{CO}_{2}$ which mainly include the following aspects: (1) Carbon emissions estimates. Most scholars calculate the carbon emission based on the carbon emission 
coefficient method (Ning and Zhang, 2014; Gong, 2015), and some scholars estimate the carbon emission amount through the indirect calculation method. The input-output method is mostly used for the calculation of implied carbon dioxide (He, 2012; Zhao et al., 2019). Due to the input-output method's lack of accuracy, the life cycle method is also favored by many scholars (Xia et al., 2010; Cao et al., 2011). (2) Identification of driving factors. With the help of LMDI decomposition method, many scholars can calculate the driving direction and contribution value of each factor ( $\mathrm{Li}$ and Zhang, 2016; Sun and Zhou, 2017), and some scholars use Stochastic Impacts by Regression on Population, Affluence, and Technology (STIRPAT) model to conduct regression analysis and calculate the regression coefficient of driving factors. The direction and magnitude are judged by the positive and negative values of the coefficients (Feng et al., 2019). In addition, some scholars use the Kaya identity to analyze (Pan and $\mathrm{Hu}$, 2019; Wang and He, 2018). (3) Study on the potential of reducing carbon emission. There are many theoretical analyses on the potential of carbon emission reduction (Tian and Ding, 2018; Zhang et al., 2015). Some scholars use the model to quantify the potential of carbon emission reduction, such as using decoupling index to quantify the potential of carbon emission reduction into elasticity index (Wang and Zhu, 2018; He and Cai, 2016), or different carbon emission scenarios are respectively set to predict the value based on the regression coefficients and the difference between each prediction result is calculated ( $\mathrm{Li}$ et al., 2018; Gu et al., 2013). In view of the existing research, the research methods on carbon emission have been more comprehensive, but most of the research objects are based on the national or provincial panel data, while the literature on urban agglomerations for the early stage is less. Therefore, the carbon emission of Circum-Changsha-Zhuzhou-Xiangtan urban agglomeration is estimated from three perspectives such as agriculture, industry and transportation in a "bottom-up" way. At the same time, the relationship between carbon emission and GDP per capita is fitted, and the factors that drive the change of urban carbon emission are decomposed through LMDI model. Finally, the decoupling elasticity is established to analyze the dependence between urban carbon emission and economic development, so as to find a more suitable emission reduction path for the development of Circum-Changsha-ZhuzhouXiangtan urban agglomeration, and provide more scientific policy suggestions for its green, coordinated and sustainable development.

\section{Materials and methods}

\section{Method for estimating carbon emissions}

In this paper, the "bottom-up" method is used to calculate the emissions of $\mathrm{CO}_{2}$ in the primary industry (agriculture), the secondary industry (industry) and the tertiary industry (transportation (the main carbon source)) and summarize to obtain the emissions of $\mathrm{CO}_{2}$ in the Circum-Changsha-Zhuzhou-Xiangtan urban agglomeration of China. The detailed calculation ideas are as follows:

$$
C_{\mathrm{tj}}=C_{A t j}+C_{I t j}+C_{T t j}
$$

where $C_{t j}$ indicates the emission of the $j$ city in the $t$ year, $C_{A t j}, C_{I t j}$ and $C_{T t j}$ indicate the emissions of $\mathrm{CO}_{2}$ in the $j$ city in the $t$ year from agriculture, industry and 
transportation. The calculation formula for carbon emissions in different industries is broken down as follows:

(1) Carbon emission from urban agriculture. The sources of carbon emission in agriculture mainly come from agricultural cultivation and fertilization. Therefore, four factors such as agricultural machinery, effective irrigation area, chemical fertilizer and rural electricity are selected to calculate the urban agricultural carbon emission. The formula is as follows:

$$
C_{A t j}=\sum_{t} F_{t i} \cdot \rho_{i}
$$

where $C_{A t j}$ indicates the carbon emission of the $j$ city in the $t$ year, $F_{t i}$ indicates the total consumption of the $i$ agricultural element in the $t$ year, and $\rho_{i}$ indicates the corresponding carbon emission factor of the $i$ element. According to the calculation of agricultural carbon emissions in Jiangxi Province (Cao and Cao, 2016), the carbon emission coefficients of agricultural machinery, effective irrigation area, fertilizer and rural electricity consumption are selected respectively as $1.8 \mathrm{t} / 103 \mathrm{kw} \cdot \mathrm{h}$, $20.476 \mathrm{t} / 103 \mathrm{~km}^{3}, 0.8956 \mathrm{t} / \mathrm{t}, 9.26 \mathrm{t} / 104 \mathrm{kw} \cdot \mathrm{h}$.

(2) Carbon emission from urban industry. Considering the availability and representativeness of the data, this paper selects the total amount of energy used by the large industrial enterprises after being converted into standard coal to calculate the urban industrial carbon emission. The calculation formula is as follows:

$$
C_{I t j}=\sum_{t} F_{t i} \cdot \rho_{i}
$$

where $C_{I t j}$ indicates the industrial carbon emission of the $j$ city in the $t$ year, $F_{t i}$ indicates the consumption of the $i$ energy in the $t$ year, and $\rho_{i}$ indicates the corresponding carbon emission factor of the $i$ energy. The carbon emission coefficient of standard coal is $2.493 \mathrm{t} / \mathrm{t}$, taken based on the IPCC China Greenhouse Gas Emission List.

(3) Carbon emission from urban transportation. According to the method of calculating the carbon emission in transportation and the applicability of the data in existing literature, this paper selects the emission of $\mathrm{CO}_{2}$ from passenger and freight vehicles $C_{T p t j}$ and residential vehicles $C_{T u t j}$ to replace the carbon emission of urban transportation. The calculation formula is as follows:

$$
C_{T t j}=\mathrm{C}_{T p t j}+\mathrm{C}_{T u t j}
$$

The carbon emissions of passenger and freight vehicles are calculated by using the calculation method of the United Nations World Tourism Organization (UNWTO), and the carbon emission of each traffic mode is estimated by the product of the turnover of each traffic mode and the corresponding carbon emission coefficient. The formula is as follows:

$$
C_{T p t j}=\sum_{t} F_{p t i} \cdot \rho_{i}
$$


where, $C_{T p t j}$ indicates the emission of $\mathrm{CO}_{2}$ from passenger and freight vehicles, $F_{p t i}$ indicates the turnover of the $i$ traffic mode in the $t$ year, and $\rho_{i}$ indicates the corresponding carbon emission factor of different turnover modes. In view of China's lack of authoritative turnover carbon emission data, this paper determines passenger vehicle carbon emission coefficient (He, 2010; Paul and Ghislain, 2010), which is converted to a freight carbon emission factor according to the unit cargo turnover provided by Greenhouse Gas Protocol and European Chemical Industry Association. The transport modes of passenger transport and freight transport statistics are railway, highway, waterway and aviation, and the emission coefficients of passenger transport turnover are respectively $27 \mathrm{~g} / \mathrm{p} \cdot \mathrm{km}$ and $133 \mathrm{~g} / \mathrm{p} \cdot \mathrm{km}$, the emission coefficients of freight transport turnover are respectively $28 \mathrm{~g} / \mathrm{ton} \cdot \mathrm{km}, 327 \mathrm{~g} / \mathrm{ton} \cdot \mathrm{km}, 53 \mathrm{~g} / \mathrm{ton} \cdot \mathrm{km}$ and $1,961 \mathrm{~g} / \mathrm{ton} \cdot \mathrm{km}$.

$$
C_{T u t j}=\sum_{t} G_{u t i} \times S_{u t i} \times Q_{u t i} \times J_{u t i}
$$

where, $C_{T \text { ut } j}$ indicates the emission of $\mathrm{CO}_{2}$ from residential vehicles in the $j$ city in the $t$ year, $G_{\text {uti }}$ indicates the fuel consumption of $100 \mathrm{~km}$ of the $i$ transportation mean, $S_{\text {uti }}$ indicates the annual mileage of the $i$ transportation mean, and $I_{\text {uti }}$ indicates the carbon emission factor of the $\mathrm{i}$ transportation mean. Due to the difficulty in obtaining the corresponding data on the energy consumption and mileage of residential vehicles, according to the calculation of the carbon emissions in transportation of Wuhan (Gong, 2015) and Zhengzhou (Ning, 2014) and in combination with the per capita GDP difference of different provinces, this paper estimates the average annual mileage of different residential vehicles in the cities of the the Circum-Changsha-ZhuzhouXiangtan urban agglomeration proportionally. The fuel consumption of private cars for $100 \mathrm{~km}$ is mainly $93 \#$, taking $0.1 \mathrm{~L} / \mathrm{km}$ and the motorcycles mainly 125 motorcycles taking $0.22 \mathrm{~L} / \mathrm{km}$.

\section{Drive factor decomposition model}

The LMDI decomposition method overcomes the problem that the traditional decomposition method has the residual term. Therefore, according to the analysis framework (Albrecht et al., 2002), the carbon emission of Circum-Changsha-ZhuzhouXiangtan urban agglomeration of China is decomposed as follows:

$$
C=\sum_{i}\left(\frac{C_{i}}{E_{i}} \times \frac{E_{i}}{E} \times \frac{E}{Y} \times \frac{Y}{P} \times P\right)
$$

where, $C$ indicates the total carbon emission of Circum-Changsha-Zhuzhou-Xiangtan urban agglomeration, $C_{i}$ indicates the total carbon emission of the $i$ industry, $E_{i}$ indicates the energy consumption of the $i$ industry, $E$ indicates the total energy consumption of urban agglomerations, $Y$ indicates the GDP of urban agglomeration and $P$ indicates employees in the urban agglomeration. In order to facilitate that decomposition, it is expanded to the follow form:

$$
A_{i}=\frac{C_{i}}{E_{i}}, \quad B_{i}=\frac{E_{i}}{E}, \quad D=\frac{E}{Y}, \quad I=\frac{Y}{P}, \quad K=P
$$


$\mathrm{Ai}, \mathrm{Bi}, \mathrm{D}, \mathrm{I}$ and $\mathrm{K}$ respectively indicate carbon emission coefficient effect, industrial structure effect, energy intensity effect, economic development effect and human capital effect.

If the total carbon emission of urban agglomerations in the basic period is set as $C^{0}$, after $T$ period, the change in the total carbon emission of urban agglomeration can be expressed as:

$$
\begin{aligned}
& \Delta C=C^{T}-C^{O} \\
& =\Delta C_{A i}+\Delta C_{B i}+\Delta C_{D}+\Delta C_{I}+\Delta C_{K}
\end{aligned}
$$

Considering that the energy carbon emission coefficient is constant, $\Delta C_{A i}$ is not considered in actual calculation. According to the study (Ang et al., 1998), the expression of contribution value of each element can be further expressed as

$$
\begin{gathered}
\Delta C_{B i}=\sum_{i}\left(\frac{C_{i}^{T}-C_{i}^{0}}{\ln C_{i}^{T}-\ln C_{i}^{0}}\right) \times \ln \left(\frac{B_{i}^{T}}{B_{i}^{0}}\right) ; \Delta C_{D}=\sum_{i}\left(\frac{C_{i}^{T}-C_{i}^{0}}{\ln C_{i}^{T}-\ln C_{i}^{0}}\right) \times \ln \left(\frac{D^{T}}{D^{0}}\right) \\
\Delta C_{I}=\sum_{i}\left(\frac{C_{i}^{T}-C_{i}^{0}}{\ln C_{i}^{T}-\ln C_{i}^{0}}\right) \times \ln \left(\frac{I^{T}}{I^{0}}\right) ; \Delta C_{K}=\sum_{i}\left(\frac{C_{i}^{T}-C_{i}^{0}}{\ln C_{i}^{T}-\ln C_{i}^{0}}\right) \times \ln \left(\frac{K^{T}}{K^{0}}\right)
\end{gathered}
$$

\section{Decoupling elasticity coefficient}

According to the explanation of the decoupling analysis elasticity (Tapio, 2005), the decoupling elasticity coefficient can be expressed as:

$$
e_{j}=\frac{\Delta C_{j} / C_{j}}{\Delta G D P_{j} / G D P_{j}}
$$

where, $e_{j}$ indicates the decoupling elasticity coefficient of the $j$ city, and $C_{j}$ indicates the total carbon emission of the $j$ city. The different elasticity magnitudes represent different decoupling states, and the specific corresponding relationship is shown in Table 1 .

Table 1. Type of Tapio decoupling elasticity

\begin{tabular}{c|c|c|c|c}
\hline \multicolumn{2}{c|}{ Status } & $\begin{array}{c}\text { Environmental } \\
\text { pressure }\end{array}$ & $\begin{array}{c}\text { Economic } \\
\text { growth }\end{array}$ & Elasticity \\
\hline \multirow{3}{*}{$\begin{array}{c}\text { Negative } \\
\text { decoupling }\end{array}$} & Weak negative decoupling & $<0$ & $<0$ & $0 \leq \mathrm{t}<0.8$ \\
& Strong negative decoupling & $>0$ & $<0$ & $\mathrm{t}<0$ \\
\hline \multirow{3}{*}{ Expansion negative decoupling } & $>0$ & $>0$ & $\mathrm{t}>1.2$ \\
\hline \multirow{3}{*}{ Connection } & Decline decoupling & $<0$ & $<0$ & $\mathrm{t}>1.2$ \\
& Strong decoupling & $<0$ & $>0$ & $\mathrm{t}<0$ \\
& Weak decoupling & $>0$ & $>0$ & $0 \leq \mathrm{t}<0.8$ \\
\hline
\end{tabular}




\section{Data sources}

This paper chooses Circum-Changsha-Zhuzhou-Xiangtan urban agglomeration of China as the research object, which includes the core area (Changsha, Zhuzhou, and Xiangtan); and radiation area (Hengyang, Yueyang, Changde, Yiyang and Loudi). The time span of data collection is from 2008 to 2016. The relevant data are mainly from the Statistical Yearbook of Hunan Province, the Statistical Yearbook of Chinese Cities and the Statistical Annual Report published by the statistical offices of each city from 2009 to 2017.

\section{Results and discussion}

\section{Estimation results of carbon emission from Circum-Changsha-Zhuzhou-Xiangtan} urban agglomeration of China

Carbon emission and carbon emission intensity of Circum-Changsha-ZhuzhouXiangtan urban agglomeration of China from 2008 to 2016 are shown in Table 2. As can be seen in Figure 1, the carbon emission of the Circum-Changsha-ZhuzhouXiangtan urban agglomeration from 2008 to 2016 can be divided into two phases. The first phase, 2008-2011, shows a steady upward trend in total carbon emissions at a relatively high growth rate, with the highest growth rate $(15.18 \%)$ in 2011 , increasing from $26.21 \times 10^{7}$ tons in 2010 to $30.19 \times 10^{7}$ tons in 2011 , and the carbon emissions of the three major industries also increased rapidly in this period. In the second phase, 2011-2016, the total carbon emissions fluctuated slightly, with positive and negative growth alternately. The industrial carbon emission fell several times at this phase. Except for a sharp rise of the carbon emission due to the large amount of fertilizer used for the flood in 2013, it showed a slow growth in all other times. The carbon emission in transportation industry after 2011 had a lower growth rate.

Table 2. Carbon emissions of Circum-Changsha-Zhuzhou-Xiangtan urban agglomeration in 2008-2016

\begin{tabular}{c|c|c|c|c|c}
\hline \multirow{2}{*}{ Year } & \multicolumn{3}{|c|}{ Carbon emissions of the Changsha, Zhuzhou and Xiangtan urban } & \\
\cline { 2 - 4 } & $\begin{array}{c}\text { Agglomerations (10,000 tons) } \\
\text { Agricultural } \\
\text { carbon } \\
\text { emissions }\end{array}$ & $\begin{array}{c}\text { Industrial } \\
\text { carbon } \\
\text { emissions }\end{array}$ & $\begin{array}{c}\text { Transportation } \\
\text { carbon } \\
\text { emissions }\end{array}$ & $\begin{array}{c}\text { Total carbon } \\
\text { emissions }\end{array}$ & $\begin{array}{c}\text { Carbon intensity } \\
\text { (ton/10,000 yuan) }\end{array}$ \\
\hline 2008 & 670 & 19093 & 1681 & 21444 & 2.45 \\
2009 & 765 & 20899 & 2196 & 23860 & 2.31 \\
2010 & 795 & 22569 & 2849 & 26213 & 2.09 \\
2011 & 877 & 25805 & 3509 & 30191 & 1.95 \\
2012 & 880 & 24658 & 4548 & 30086 & 1.70 \\
2013 & 1326 & 25322 & 5214 & 31862 & 1.62 \\
2014 & 986 & 23932 & 5594 & 30512 & 1.41 \\
2015 & 981 & 23006 & 6022 & 30009 & 1.28 \\
2016 & 1000 & 22956 & 6479 & 30435 & 1.19 \\
\hline
\end{tabular}




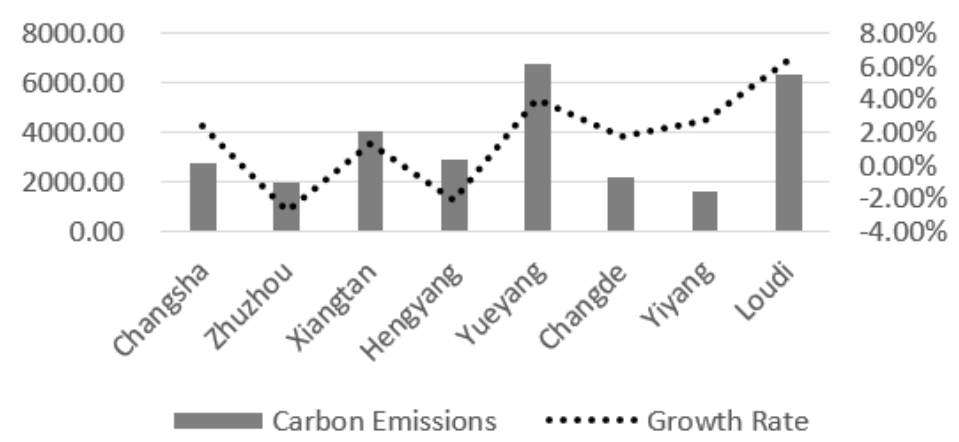

Figure 1. Carbon emissions and growth rate of Circum-Changsha-Zhuzhou-Xiangtan urban agglomeration in 2008-2016

The EKC curve (Grossman and Krueger, 1995) is used to further explore the relationship between economic development and environmental pollution. Based on the data of carbon emissions and GDP per capita from 2008 to 2016 in the CircumChangsha-Zhuzhou-Xiangtan urban agglomeration, a EKC curve regression model for the urban carbon emission including primary, secondary and tertiary terms is established:

$$
Y=\alpha_{0}+\alpha_{1} X+\alpha_{2} X^{2}+\alpha_{3} X^{3}+\varepsilon
$$

where $Y$ and $X$ respectively indicate the carbon emission and per capita GDP of the urban agglomeration, and $\alpha 0, \alpha 1, \alpha 2, \alpha 3$ and $\varepsilon$ indicate the parameters to be estimated and the random error term. The regression analysis and test are carried out by using the software of E-Views (9.0). It is found that the quadratic function fitting curve is the best, with the fitting degree of 0.971 , the $\mathrm{F}$ value is 101.75 , and the $\mathrm{P}$ values all pass the significance test. The metrology model is shown in Figure 2, the carbon emission and per capita GDP of Circum-Changsha-Zhuzhou-Xiangtan urban agglomeration shows an obvious "inverted-U” curve.

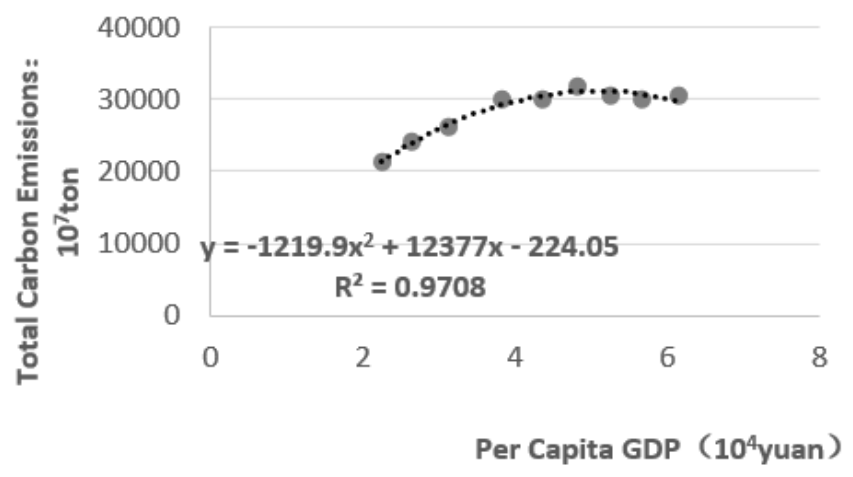

Figure 2. Curve of carbon emissions and per capita GDP Circum-Changsha-Zhuzhou-Xiangtan urban agglomeration in 2008-2016

According to the derivation of the fitting curve, the stagnation point is 5.073, that is, when the per capita GDP exceeds 5.073, the carbon emission is likely to fall, which 
may be due to complicated reasons: for example, the vigorous development of heavy industry in the early period of the economic downturn to rapidly improve the economic level has led to increased pollution. As the environment deteriorates and resource scarcity increases, the public has paid more attention to energy conservation and emission reduction and accelerated the research and development of high-tech and environmentally-friendly technology products, so the urban carbon emission has in turn been controlled.

\section{Driving factor decomposition results}

The LMDI decomposition results are shown in Table 3, as can be seen in conjunction with the judgement matrix (Table 4). During 2008-2016, the carbon emission in the Circum-Changsha-Zhuzhou-Xiangtan urban agglomeration of China increased 5 times and decreased 3 times, industrial structure and energy intensity suppressed the carbon emission of the urban agglomeration, and the economic development and human capital played an important role. Among them, the economic effect is the most remarkable, and its annual contribution to the growth of the carbon emission in the Circum-ChangshaZhuzhou-Xiangtan urban agglomeration was $87 \%$, so the increase of carbon emission in the urban agglomeration mainly depended on the driving value of economic development. The negative driving effect of industrial structure was obvious from 2009 to 2012, but the driving effect of energy intensity from 2012 to 2016 was obviously higher than that of industrial structure, and the contribution value increased rapidly. The growth in carbon emission in the urban agglomeration slowed down after 2013.

Table 3. Driving factors decomposition of carbon emissions of Circum-Changsha-ZhuzhouXiangtan urban agglomeration in 2008-2016

\begin{tabular}{c|c|c|c|c|c}
\hline Year & $\Delta C$ & $\Delta C_{B i}$ & $\Delta C_{D}$ & $\Delta C_{I}$ & $\Delta C_{K}$ \\
\hline 2009 & 5.58 & -19.43 & -9.62 & 28.94 & 5.69 \\
2010 & 22.37 & -37.08 & -23.88 & 72.57 & 10.76 \\
2011 & 63.57 & -62.61 & -23.08 & 129.64 & 19.61 \\
2012 & 49.13 & -74.66 & -63.86 & 164.11 & 23.55 \\
2013 & 57.09 & -81.65 & -84.97 & 195.47 & 28.25 \\
2014 & 36.23 & -83.85 & -122.35 & 212.82 & 29.61 \\
2015 & 34.09 & -75.60 & -153.83 & 228.54 & 34.98 \\
2016 & 41.86 & -73.85 & -174.31 & 249.76 & 40.27
\end{tabular}

Table 4. Driver factors judgment matrix of Circum-Changsha-Zhuzhou-Xiangtan urban agglomeration in 2008-2016

\begin{tabular}{c|c|c|c|c|c}
\hline Period & $\Delta C$ & $\Delta C_{B i}$ & $\Delta C_{D}$ & $\Delta C_{I}$ & $\Delta C_{K}$ \\
\hline $2008-2011$ & $\uparrow$ & - & - & + & + \\
$2011-2012$ & $\downarrow$ & - & - & + & + \\
$2012-2013$ & $\uparrow$ & - & - & + & + \\
$2013-2015$ & $\downarrow$ & - & - & + & + \\
$2015-2016$ & $\uparrow$ & - & - & + & + \\
& & Not obvious & Obvious & Obvious & Not obvious \\
\hline
\end{tabular}


From the time series, the contribution value of each factor is increasing year by year. Besides the negative growth of industrial structure after 2014, there is still room for adjustment. The growth rate of the contribution of the four driving factors to the urban carbon emission has gradually slowed down, but the growth rate gap is relatively obvious, of which the energy intensity contribution value has the fastest growth, with an average annual growth rate of $62.52 \%$. Therefore, in 2013, its contribution value quickly exceeded the industrial structure and gradually had a gap, effectively suppressing the increase of carbon emissions of the urban agglomeration, which indicates that giving enough attention to energy consumption can effectively promote the process of green development.

\section{Decoupling analysis}

From Table 5, the overall results show that the decoupling elasticity of the CircumChangsha-Zhuzhou-Xiangtan urban agglomeration of China was mainly declining, with strong decoupling in 2011-2012, 2013-2014 and 2014-2015, that is, the carbon emission is decreasing with the economic growth, but weak decoupling in the rest of the years, which indicates that the overall economic growth rate is slightly higher than the carbon emission growth rate, and there is a strong decoupling trend.

Table 5. Decoupling status of Circum-Changsha-Zhuzhou-Xiangtan urban agglomeration in 2008-2016

\begin{tabular}{c|c|c|c|c|c|c|c|c|c}
\hline City & E/S & $\mathbf{2 0 0 8 - 2 0 0 9}$ & $\mathbf{2 0 0 9 - 2 0 1 0}$ & $\mathbf{2 0 1 0 - 2 0 1 1}$ & $\mathbf{2 0 1 1 - 2 0 1 2}$ & $\mathbf{2 0 1 2 - 2 0 1 3}$ & $\mathbf{2 0 1 3 - 2 0 1 4}$ & $\mathbf{2 0 1 4 - 2 0 1 5}$ & $\mathbf{2 0 1 5 - 2 0 1 6}$ \\
\hline Chang & E & 0.6048 & 0.4357 & 0.5588 & 0.1536 & 0.6658 & -0.3655 & 0.2734 & 1.4824 \\
Sha & S & W.D & W.D & W.D & W.D & W.D & S.D & W.D & E.N.D \\
Zhu & E & 0.3767 & 0.6397 & 0.0470 & 0.0796 & 0.4271 & -1.1267 & -1.3301 & 0.5989 \\
Zhou & S & W.D & W.D & W.D & W.D & W.D & S.D & S.D & W.D \\
Xiang & E & 1.7249 & 0.0253 & 0.5722 & -0.4813 & 0.0212 & -0.6897 & -1.3624 & -0.0452 \\
Tan & S & E.N.D & W.D & W.D & S.D & W.D & S.D & S.D & S.D \\
Heng & E & 1.0532 & 0.6965 & 0.7723 & -0.9595 & 0.1059 & -1.2691 & -1.0552 & -0.1453 \\
Yang & S & E.C & W.D & W.D & S.D & W.D & S.D & S.D & S.D \\
Yue & E & -0.1865 & 0.3940 & 1.0166 & 0.5749 & 0.6323 & -1.0038 & 0.6645 & 0.1260 \\
Yang & S & S.D & W.D & E.C & W.D & W.D & S.D & W.D & W.D \\
Chang & E & 0.7776 & 0.9752 & 0.6518 & -0.4805 & 0.9637 & -0.4340 & -0.2543 & -1.0754 \\
De & S & W.D & E.C & W.D & S.D & E.C & S.D & S.D & S.D \\
Yi & E & 0.9668 & 0.6350 & 0.7119 & -0.4823 & 0.9568 & -0.0705 & 0.2528 & 0.3337 \\
Yang & S & E.C & W.D & W.D & S.D & E.C & S.D & W.D & W.D \\
Lou & E & 1.7686 & 0.6999 & 0.6472 & 0.0717 & 0.7693 & 0.4934 & -0.3180 & 0.0324 \\
Di & S & E.N.D & W.D & W.D & W.D & W.D & W.D & S.D & W.D \\
\hline \multirow{2}{*}{ Total } & E & 0.6217 & 0.4618 & 0.6515 & -0.02482 & 0.52295 & -0.42865 & -0.1989 & 0.15586 \\
& S & W.D & W.D & W.D & S.D & W.D & S.D & S.D & W.D \\
\hline
\end{tabular}

"E"stands for Elasticity; "S" stands for Status; "W.D”stands for Weak negative decoupling; "S.D"stands for Strong decoupling; "E.N.D”stands for Expansion negative decoupling; "E.C”stands for Expansion connection

By cities of the Circum-Changsha-Zhuzhou-Xiangtan urban agglomeration of China, there have been four strong decoupling phenomena in Changde and Hengyang, in which Hengyang was in the state of expansion connection during 2008-2009, that is, its carbon emission increased faster than the economic growth. It has been in a strong decoupling state since 2013. Changsha, Zhuzhou and Xiangtan, as the core regions of the Circum- 
Changsha-Zhuzhou-Xiangtan urban agglomeration, are not in an ideal state of decoupling, and Changsha's carbon emission increased rapidly, with a negative decoupling of expansion between 2015 and 2016, that is, the growth rate of carbon emissions was far faster than the economic growth rate. In the later development, we should pay attention to the environmental governance. After 2013, Zhuzhou and Xiangtan mainly show a strong decoupling trend, that is, the correlation between economic development and carbon emission will gradually weaken, which is more conducive to energy conservation and emission reduction. The decoupling in Yiyang and Loudi was not satisfactory. The economic growth rate was slightly higher than the carbon emission growth rate, but lower than the average level of the urban agglomeration. According to the "inverted-U" curve between carbon emission and per capita GDP in Figure 2, Yiyang and Loudi are still in the increasing stage of "invertedU" curve, so the two cities should first focus on promoting the economy, only after the economic development to a certain level, is there enough funds and foundation to effectively promote energy-saving and emission reduction.

\section{Conclusions and suggestions}

In this paper, the carbon emission of the Circum-Changsha-Zhuzhou-Xiangtan urban agglomeration of China is decomposed into three parts: agriculture, industry, and transportation. At the same time, based on the panel data from 2008 to 2016, this paper uses LMDI decomposition method to calculate the direction and contribution value of the different factors that drive the carbon emission change of the urban agglomeration. Finally, the decoupling elasticity index is used to calculate the decoupling elasticity of each city and the urban agglomeration as a whole, and the following conclusions are drawn:

First of all, the annual growth rate of carbon emission of the Circum-ChangshaZhuzhou-Xiangtan urban agglomeration is decreasing gradually though its carbon emission is rising and the fluctuation of industrial carbon emission is the most obvious. Therefore, the carbon emission of the whole city has an alternatively positive and negative growth trend after 2011. According to the results of the EKC curve, it is found that the carbon emission and the per capita GDP have a relatively obvious "inverted-U" curve, that is, when the per capita GDP reaches the stagnation point of 5.073 of the curve, with the increase of per capita GDP, the carbon emission shows a downward trend instead. Second, from the driving factors, industrial structure and energy intensity have the restraining effect on the total carbon emission, while economic development and population scale have driven the growth of the carbon emission, of which the driving role of economic development is the most significant and is the main reason for the increase in the carbon emission. The restraining effect of energy intensity gradually shows up in the later period and exceeds the negative driving effect of industrial structure, as the main factor that promotes the decrease in the carbon emission intensity. Finally, the overall decoupling of the Circum-Changsha-Zhuzhou-Xiangtan urban agglomeration is good and its carbon emission growth is always weaker than economic growth in the research period. The elasticity decoupling result in Hengyang and Changde is better, and their economic development and carbon emission both show strong decoupling trend but still have great room in Loudi and Yiyang. However, in Changsha, Zhuzhou and Xiangtan, as the core region of the urban agglomeration, the effect of decoupling is not obvious though the level of the economic development is 
relatively high, and the economic development is only slightly faster than the growth of carbon emission. In the later development, more emphasis should be placed on energy conservation and emission reduction

In the future research, there is still something worthy of further exploration. First of all, for the research objectives, this paper only studies the Circum-Changsha-ZhuzhouXiangtan urban agglomeration, which includes eight cities, the results are not universal; the range of the research objectives can be expended in the future. And then, due to the lack of some data, the calculation of carbon emissions is not comprehensive, for example, date of agricultural film and pesticides have not found. Finally, for the driving factors of carbon emissions, this paper only chooses the most significant four factors according to the research of a large amount of literatures, which can be extended for more measurement. Under such circumstances, this paper can only be regarded as a rough evaluation and preliminary study on the carbon emissions of the CircumChangsha-Zhuzhou-Xiangtan urban agglomeration. As for the more in-depth content and more precise conclusions, it needs to be enriched in the future research. At the same time, I also hope that the research in this paper can be used as a reference for the research work of other scholars, and can provide useful thinking for the green development and practical work of urban agglomerations.

Acknowledgements. The authors would like to thank the referee for his helpful advice and comments. This is a project supported by Hunan Provincial Association of Social Sciences think tank project (ZK2019031) and Scientific Research Fund of Hunan Provincial Education Department (16A079).

\section{REFERENCES}

[1] Albrecht, J., François, D., Schoors, K. (2002): A Shapley decomposition of carbon emissions without residuals. - Energy Policy 30(9): 727-736.

[2] Cao, H. J., Song, S. L., Du, Y. B., Chen, P. (2011): Evaluation method and application for carbon emissions of machine tool based on life cycle assessment. - Computer Integrated Manufacturing Systems 17(11): 2432-243.

[3] Cao, J. W., Cao, L. J. (2016): Research on measurement and effecting factors of agricultural carbon emission in Jiangxi province. - Ecological Economy 32(7): 66$68+167$.

[4] Feng, J., Zhang, S., Wang, T. (2019): Analysis of China's interprovincial land use carbon emissions and its influencing factors. - Statistics and Decision 35(5): 141-145.

[5] Gong, Y. Y. (2015): Carbon emission calculation and influential factor decomposition of the transportation sector in Wuhan city. - China Population, Resources and Environment 25(S1): 470-474.

[6] Grossman, G. M., Krueger, A. B. (1995): Economic growth and environment. - The Quarterly Journal of Economic 110(2): 353-377.

[7] Gu, B. H., Tan, X. C., Chi, H., Wang, Y. Y. (2013): A carbon dioxide reduction potential model for chemical industry. - Chinese Journal of Management Science 21(5): 141-148.

[8] He, J. C., Li, Y. Z. (2016): Estimation of $\mathrm{CO}_{2}$ emission of locomotives in China during 1975-2005. - Advances in Climate Change Research 6(1): 35-39.

[9] He, Y., Cai, M. T. (2016): Decoupling relationship between economic growth and resource environment in Beijing-Tianjin-Hebei region. - Journal of Beijing Institute of Technology (Social Sciences) 18(5): 33-41.

[10] He, Y. Q. (2012): The measurement and application of industry complete carbon emissions. - Statistical Research 29(3): 67-72. 
[11] Li, J., Guo, J., Yuan, Q. M. (2018): Forecast of energy demand and policy impact under the background of the coordinated development in Beijing-Tianjin-Hebei Region. Journal of Arid Land Resources \& Environment 32(5): 5-11.

[12] Li, Y. M., Zhang, Y. G. (2016): Spatial decomposition analysis of factors affecting China's carbon emissions. - Journal of China University of Geosciences (Social Science) 16(3): 73-85.

[13] Ning, X. J., Zhang, J. P. (2014): Spatial and temporal characteristics of carbon emissions from urban resident travel in Zhengzhou. - Resource Science 36(5): 1021-1028.

[14] Pan, W., Hu, C. (2019): Study on carbon emissions decomposition of energy consumption in different industries in China. - Statistics and Decision 35(4): 141-145.

[15] Peeters, P., Dubois, G. (2010): Tourism travel under climate change mitigation constraints. - Journal of Transport Geography 18(3): 447-457.

[16] Sun, Y. F., Zhou, M. (2017): Decoupling and driving factors analysis between the energy carbon emissions and economic growth in China. - Review of Economy and Management 33(6): 21-30.

[17] Tapio, P. (2005): Towards a theory of decoupling: degrees of decoupling in the EU and the case of road traffic in Finland between 1970 and 2001. - Transport Policy 12(2): 137151.

[18] Tian, H., Ding, C. A. (2018): The spatial and temporal differences of carbon emissions in regional Tourism - a case study of Shandong province. - Journal of Beijing Institute of Technology (Social Science) 20(6): 45-54.

[19] Wang, J., Zhu, C. Z. (2018): international comparison of decomposition factors and decoupling effects of agricultural carbon emissions. - Statistics and Decision 34(11): 104-108.

[20] Wang, Y. B., He, Y. P. (2018): Study on greenhouse gas emission from urban waste disposal and influence mechanism. - Journal of Xi'an Jiaotong University (Social Sciences) 38(1): 60-70.

[21] Xia, D. J., Ren, Y. L., Shi, L. F. (2010): Measurement of life-cycle carbon equivalent emissions of coal-energy chain. - Statistical Research 27(8): 82-89.

[22] Zhang, Y., Xiong, X. P., Kang, Y. B. (2015): Study on influencing factors and carbon emission reduction pathway for transportation sector of China. - Environmental Protection 43(11): 54-57.

[23] Zhao, Y. W., Zuo, H. B., She, X. F., Wang, G., Xue, Q. G., Wang, J. S. (2019): Case study on calculation method of carbon dioxide emission in iron and steel industry. Nonferrous Metals Science and Engineering 10(1): 34-40. 